\title{
Oligodontia-cancer predisposition syndrome
}

INSERM

\section{Source}

INSERM. (1999). Orphanet: an online rare disease and orphan drug data base.

Oligodontia-cancer predisposition syndrome. ORPHA:300576

Oligodontia-cancer predisposition syndrome is a rare, genetic, odontologic disease characterized by congenital absence of six or more permanent teeth (excluding the third molars) in association with an increased risk for malignancies, rang ing from gastrointestinal polyposis to early-onset colorectal cancer and/or breast cancer. Ectodermal dysplasia (manifesting with sparse hair and/or eyebrows) may also be associated. 